K. Al Majali • M. B. Cooper • B. Staels • G. Luc •

M-R. Taskinen · D. J. Betteridge

\title{
The effect of sensitisation to insulin with pioglitazone on fasting and postprandial lipid metabolism, lipoprotein modification by lipases, and lipid transfer activities in type 2 diabetic patients
}

Received: 23 March 2005 / Accepted: 4 October 2005 / Published online: 21 January 2006

C) Springer-Verlag 2006

\begin{abstract}
Aims/hypothesis: Insulin resistance is thought to be central to the pathogenesis of diabetic dyslipidaemia. We hypothesised that improving insulin sensitivity would improve fasting and postprandial triglyceride metabolism in patients with type 2 diabetes. To this aim we studied fasting and postprandial lipaemia in type 2 diabetic patients before and after sensitisation to insulin with pioglitazone, compared with that observed in patients on an insulinproviding regime. Methods: In a double-blind placebo-controlled protocol, 22 patients with type 2 diabetes were randomly allocated to receive either pioglitazone ( $45 \mathrm{mg} /$ day) or glibenclamide ( $5 \mathrm{mg} /$ day), for a 20 -week period. Fasting and postprandial lipid metabolism were investigated at baseline and at the end of the treatment period. A group of non-diabetic subjects was also studied. Results: Compared with glibenclamide treatment, pioglitazone treatment de-
\end{abstract}

This study was funded by Takeda Europe, UK, in the form of an MD studentship award to K. Al-Majali. D. J. Betteridge has sat on advisory boards and lectured at meetings sponsored by Takeda. M.-R. Taskinen has received honoraria from Takeda. The study was conceived and conducted by the investigators who had complete control of the data, analyses and the preparation of this article.

K. A. Majali · M. B. Cooper $(\bowtie) \cdot$ D. J. Betteridge

Royal Free and University College Medical School,

Department of Medicine,

5th Floor Jules Thorn Institute,

Middlesex Hospital, Mortimer St,

London W1N 8AA, UK

e-mail: michael.cooper@ucl.ac.uk

Tel.: +44-020-76799196

Fax: +44-020-73089117

B. Staels · G. Luc

UR 545 INSERM, Atherosclerosis Department,

Pasteur Institute of Lille, and the Faculty of Pharmacy,

Lille2 University,

Lille, France

M.-R. Taskinen

Helsinki University Hospital,

Biomedicum,

Helsinki, Finland creased fasting triglyceride, glucose and insulin levels and the homeostasis model assessment score of insulin resistance. Decreased fasting triglyceride after pioglitazone treatment was due to reduced VLDL triglyceride, particularly VLDL-2. Lipoprotein lipase activity was unchanged by pioglitazone treatment but hepatic lipase showed a significant decrease. Pioglitazone treatment lowered total postprandial triglyceride, as well as chylomicron- and chylomicron-remnant retinyl palmitate levels to normal. Glucose disposal improved but remained abnormal. Conclusions/interpretation: Insulin sensitisation with pioglitazone has major effects in restoring postprandial lipaemia to normal, while also correcting fasting hypertriglyceridaemia; both factors may have consequences for atherogenic risk in diabetes.

Keywords Chylomicron metabolism - Glibenclamide . Insulin resistance $\cdot$ Lipase $\cdot$ Lipid transfer proteins Lipoproteins $\cdot$ Pioglitazone $\cdot$ Postprandial lipid metabolism - Thiazolidinedione - Type 2 diabetes

Abbreviations AIP: atherogenic index of plasma - CETP: cholesteryl ester transfer protein - FPG: fasting plasma glucose - HOMA: homeostasis model assessment - LCAT: lecithin:cholesterol acyl transferase - LPL: lipoprotein lipase - PLTP: phospholipid transfer protein - PPAR: peroxisome proliferator-activated receptor $\cdot \mathrm{RP}$ : retinyl palmitate $\cdot S_{\mathrm{f}}$ : Svedberg flotation rate $\cdot$ TZD: thiazolidinedione

\section{Introduction}

Dyslipidaemia is common in type 2 diabetes and contributes to an increased risk of atherosclerosis-related disease. It is characterised by fasting hypertriglyceridaemia, low HDL cholesterol, the accumulation of triglyceride-rich remnant particles and a shift in the size distribution of LDL to a preponderance of smaller, denser particles [1-3]. Abnormal postprandial lipid metabolism is also an important feature of diabetic dyslipidaemia and contributes 
to the qualitative and quantitative lipid and lipoprotein abnormalities observed in the fasting state [4-6].

In insulin resistance, postprandial VLDL secretion is not suppressed. As a result, VLDL competes with the alimentary lipoproteins for the available lipoprotein lipase (LPL) activity needed to produce remnant particles destined for hepatic uptake along with intermediate-density lipoprotein, the precursor of LDL [7]. Along with prolonged lipaemia, abnormal lipid exchange mediated by specific transfer proteins leads to formation of triglyceride-rich LDL and HDL [8]. These are substrates for hepatic lipase, the activity of which is increased in insulin resistance and generates atherogenic small dense LDL and HDL species that, together with prolonged postprandial lipaemia itself, have been shown to be cardiovascular disease risk factors $[9,10]$.

Insulin resistance is thought to be central to the pathogenesis of diabetic dyslipidaemia. We hypothesised that improving insulin sensitivity would improve fasting and postprandial triglyceride metabolism in patients with type 2 diabetes. We studied the effect of a thiazolidinedione (TZD), pioglitazone, on fasting triglyceride concentrations, postprandial lipaemia and glucose metabolism in patients with type 2 diabetes. The TZDs are a novel class of drug that act by making peripheral tissues more responsive to insulin $[11,12]$. They are agonists for the peroxisome proliferator-activated receptor (PPAR)- $\gamma$, one of a family of nuclear receptors concerned with metabolic fuel selection and storage [13]. We incorporated a control patient group randomised to receive insulin-providing therapy with glibenclamide, which does not to affect insulin resistance or postprandial lipid metabolism $[14,15]$. This was administered at a low dose in order to achieve glycaemic control similar to that of the pioglitazone group, as assessed by $\mathrm{HbA}_{1} \mathrm{c}$. We also studied a group of nondiabetic subjects with the aim of assessing to what extent TZD treatment restored lipid and lipoprotein metabolism to normal.

\section{Subjects and methods}

\section{Subjects}

Twenty-two patients with type 2 diabetes and previously identified as moderately hypertriglyceridaemic (triglyceride $>1.7 \mathrm{mmol} / \mathrm{l}$ but $<5 \mathrm{mmol} / \mathrm{l}$ ) with an $\mathrm{HbA}_{1} \mathrm{c}<8.5 \%$ achieved with sub-maximum doses of single oral hypoglycaemic agents were recruited from the diabetic clinic at the Middlesex Hospital London. No patients had prior treatment with TZD or were receiving lipid-lowering therapy. Subjects with abnormal hepatic function, abnormal renal function or other conditions known to affect lipid metabolism, or who consumed excessive amounts of alcohol, were excluded from the study. Female diabetic subjects were postmenopausal and none was taking hormonal therapy. Ten healthy controls were also recruited, none of whom was taking medication or had any family history of type 2 diabetes.
Study design

Current oral hypoglycaemic therapy was withdrawn for 4 weeks, during which time all subjects took matching placebo tablets for pioglitazone and glibenclamide. During this time advice on diet and lifestyle measures was reinforced. At the completion of this stabilisation phase (baseline) patients received a test meal and post-heparin plasma was obtained for measurement of hepatic and LPL activities. Patients were randomised to receive either pioglitazone $(45 \mathrm{mg})$ or glibenclamide $(5 \mathrm{mg})$ in a double-blind placebo-controlled protocol designed to maintain glycaemic control at the baseline level for 20 weeks. Subjects were monitored regularly for glycaemic control. At the completion of the treatment period, lipase activities were measured and subjects received another test meal identical to the first. Control individuals received no treatment prior to the test meal. The local ethics committee approved the study and all subjects gave fully informed written consent for the study, which was undertaken in accordance with the Declaration of Helsinki.

\section{Test meal}

After a $14 \mathrm{~h}$ fast, patients and control subjects were given a standard mixed meal with a high fat content consisting of $110 \mathrm{~g}$ fat (polyunsaturated : saturated fat ratio $=0.4$ ), $46 \mathrm{~g}$ protein, $70 \mathrm{~g}$ complex carbohydrate and $0.74 \mathrm{~g}$ cholesterol served as scrambled egg made with double cream, a wholemeal bread cheese sandwich and a milkshake prepared with double cream. The meal was given at 08:00 hours following a fasting blood sample and was consumed within $15 \mathrm{~min}$. Retinyl palmitate (RP) (300,000 IU) (Arovit, Roche UK Ltd) was blended into the milkshake to label intestinally derived lipoproteins. Postprandial metabolism was monitored for $8 \mathrm{~h}$. No smoking was allowed during the test but the subjects were permitted water freely. Blood samples were collected from an indwelling catheter every $30 \mathrm{~min}$ for the first $2 \mathrm{~h}$ and then hourly for $6 \mathrm{~h}$. Postprandial excursions of triglyceride, glucose and RP were assessed by integration of the incremental responses to give the AUC.

Isolation of total VLDL, VLDL-1, VLDL-2, intermediate-density lipoprotein and LDL

Total VLDL was isolated from plasma by flotation. Samples were overlaid with saline and centrifuged for $30 \mathrm{~min}$ at $417,000 \mathrm{~g}_{\mathrm{av}}$ at $16^{\circ} \mathrm{C}$ using a high-speed ultracentrifuge (TLA-110 rotor Optima MAX; Beckman Coulter (UK) Ltd, High Wycombe, UK) [16-18]. Total VLDL was aspirated from the saline layer and the density adjusted to $1.13 \mathrm{~g} / \mathrm{ml}$ with a salt solution $(\rho=1.0852 \mathrm{~g} / \mathrm{ml})$ and overlaid with saline. Samples were centrifuged for $10 \mathrm{~min}$ at $417,000 \mathrm{~g}_{\mathrm{av}}$ at $16^{\circ} \mathrm{C}$. VLDL-1 (Svedberg flotation rate $\left.\left[S_{\mathrm{f}}\right] 60-400\right)$ migrated into the saline layer and was aspirated. The remaining sample was overlaid with 
saline and centrifuged for $40 \mathrm{~min}$ at $417,000 \mathrm{~g}_{\mathrm{av}}$ at $16^{\circ} \mathrm{C}$ and VLDL-2 $\left(S_{\mathrm{f}} 20-60\right)$ harvested from the saline layer. Intermediate-density lipoproteins $(\rho=1.019)$ and LDL $(\rho=1.063)$ were isolated from the plasma infranatant remaining after removal of total VLDL by sequential isopycnic centrifugation [19].

Isolation of chylomicrons and chylomicron remnants

Intact chylomicrons $\left(S_{\mathrm{f}}>400\right)$ were separated by flotation. Plasma was overlaid with saline and centrifuged for $7 \mathrm{~min}$ at $417,000 g_{\text {av }}$ at $16^{\circ} \mathrm{C}$. Intact chylomicrons were collected from the saline layer and stored in the dark at $-85^{\circ} \mathrm{C}$ for RP assay. Chylomicron-remnant-containing fractions $\left(S_{\mathrm{f}} 20\right.$ 400) were prepared from the infranatant by overlaying with saline and centrifugation at $417,000 g_{\text {av }}$ at $16^{\circ} \mathrm{C}$ for $30 \mathrm{~min}$. Chylomicron remnants in the saline layer were aspirated and stored in the dark at $-85^{\circ} \mathrm{C}$ for assay of RP.

\section{Measurement of HDL}

Plasma was centrifuged at $200,000 g_{\text {av }}$ at $16^{\circ} \mathrm{C}$ for $45 \mathrm{~min}$ to remove triglyceride-rich lipoproteins. HDL in the infranatant was determined by the polyethylene glycol technique (Quantolip HDL; Technoclone, UK) [20].

\section{Measurement of RP}

RP in chylomicron and chylomicron remnants was extracted with chloroform/methanol using retinyl acetate as internal standard [21]. Isocratic HPLC chromatography was carried out with a 3- $\mu$ octadecyl reversed phase column (Phenomenex, UK) using methanol as eluent. RP was detected in the effluent at $580 \mathrm{~nm}$ and the concentration calculated by comparison of the peak heights of the recovered internal standard and those of a standard solution containing retinyl acetate and RP.

Cholesterol, glucose, triglyceride and NEFA determinations

Cholesterol, glucose, triglyceride and NEFA measurements were made using spectrophotometric reagent kits (cholesterol, triglyceride and glucose: Sigma Infinity reagents, Sigma Chemical Company, Poole, Dorset, UK; NEFA: Roche Diagnostics, Lewes, East Sussex, UK).

Assay of plasma lecithin:cholesterol acyl transferase (LCAT) and cholesteryl ester transfer protein (CETP) activities

LCAT and CETP activities in the fasting and postprandial state were measured as described previously [22]. In brief, LCAT activity was measured as the decrease in plasma-free cholesterol determined hourly over $3 \mathrm{~h}$ while CETP activity was measured, under conditions where LCAT was inhibited, as the decrease in the cholesteryl ester content of HDL over $3 \mathrm{~h}$.

Determinations of phospholipid transfer protein activity (PLTP)

PLTP activity was measured in plasma as the transfer of radiolabelled phosphatidylcholine from $\left[{ }^{14} \mathrm{C}\right]$ phosphatidylcholine liposomes to an excess of exogenous human $\mathrm{HDL}_{3}$ [23].

Preparation of post-heparin plasma and assay of hepatic and LPL activities

Post-heparin plasm for assay of hepatic and LPL was prepared 1 week prior to each test meal. Subjects received an injection of $60 \mathrm{kU} / \mathrm{kg}$ heparin i.v. After $15 \mathrm{~min}$, venous blood was sampled and post-heparin plasma prepared by centrifugation; aliquots were immediately frozen and stored at $-85^{\circ} \mathrm{C}$. Hepatic lipase and LPL activities were measured by a radiometric technique involving the cleavage of $\left[{ }^{3} \mathrm{H}\right]$ oleic acid from $\left[{ }^{3} \mathrm{H}\right]$ tri-oleoyl glycerol [24].

Homeostasis model assessment score of insulin resistance and the atherogenic index of plasma (AIP)

The homeostasis model assessment (HOMA) estimate of insulin resistance was calculated from fasting plasma glucose (FPG) and insulin levels [25]. The AIP is a novel index that correlates well with the presence of small dense LDL particles in the plasma; it was calculated from the formula $\mathrm{AIP}=\log _{10}([$ triglyceride $] /[\mathrm{HDL}$ cholesterol] $)$, in which both triglyceride and HDL cholesterol are expressed as $\mathrm{mmol} / \mathrm{l}[26]$.

\section{Statistical methodology}

The significance of the effects of the treatment was obtained by paired $t$-test analyses. Differences between control subjects and the patients before and after each treatment were assessed by ANOVA with Bonferroni correction. Correlations were obtained by linear regression. Triglyceride data were $\log _{10}$ transformed before statistical analyses [27]. Analyses were made using SPSS and the level of statistical significance was set to $p \leq 0.05$.

\section{Results}

Subjects

Patient groups were well-matched at baseline (Table 1). No statistically significant differences were found between the 
diabetic patients and the control subjects with respect to weight, BMI and age. Pioglitazone and glibenclamide treatments maintained similar glycaemic control over the study period with no significant changes in $\mathrm{HbA}_{1} \mathrm{c}$. FPG was the same in both groups of patients at baseline but higher than in controls. Pioglitazone treatment caused a significant reduction in FPG not seen with glibenclamide treatment. Pioglitazone caused a significant fall in plasma triglyceride to a level that was not significantly different from control. No significant changes were observed in fasting NEFA levels, although there was a trend to a reduction in the pioglitazone group.

Pioglitazone treatment caused a decrease in fasting plasma insulin to levels seen in controls. Insulin levels were increased by glibenclamide treatment. Glibenclamide treatment caused an increase in the HOMA score, whereas pioglitazone caused a significant reduction, although it remained greater than in controls. The group receiving pioglitazone had a significant reduction in the AIP (baseline: 0.14 ; treated: $0.01, p<0.01$, paired $t$-test) that was not seen in the glibenclamide group (baseline: 0.09 ; treated: $0.23, p=0.40$ ).

Fasting plasma lipoprotein profiles

Fasting plasma lipoprotein profiles are shown in Table 2. Total VLDL cholesterol was similar in the control and the diabetic patients at baseline and did not change after treatment with pioglitazone or glibenclamide. However, total VLDL triglyceride was significantly greater in both groups of untreated diabetic patients than in the controls. Pioglitazone treatment significantly reduced total VLDL triglyceride to a level that no longer differed from that of controls.

VLDL-1 and VLDL-2 triglyceride concentrations in the diabetic patients were significantly higher than in controls. Pioglitazone treatment caused a reduction in both VLDL-1 and VLDL-2 triglyceride, but this achieved statistical significance only in the smaller VLDL-2 $(p<0.04)$, whereas
Table 1 Demographics and clinical characteristics of the study groups
Data are means \pm SEM or medians (95\% CIs) except for the duration of diabetes, which is given as means (range); $* p<0.05$, ANOVA, for difference between diabetic patients and control subjects; $\uparrow p<0.05$, paired $t$-test, for difference between baseline and treatment in pioglitazone-treated group; $\$$, $p<0.05$ for difference between baseline and treatment in glibenclamide-treated group (paired $t$-test)

\begin{tabular}{|c|c|c|c|}
\hline & \multicolumn{2}{|c|}{ Type 2 diabetic patients } & \multirow[t]{2}{*}{ Control subjects } \\
\hline & $\begin{array}{l}\text { Glibenclamide } \\
\text { group }\end{array}$ & $\begin{array}{l}\text { Pioglitazone } \\
\text { group }\end{array}$ & \\
\hline Age (years) & $58.0 \pm 2.6$ & $56.0 \pm 3.7$ & $52.4 \pm 8.9$ \\
\hline Duration of diabetes (years) & $2.4(0.5-4.8)$ & $2.6(0.5-5.2)$ & - \\
\hline $\operatorname{Sex}(M / F)$ & $8 / 3$ & $8 / 2$ & $4 / 6$ \\
\hline Smokers & 2 & 3 & 2 \\
\hline \multicolumn{4}{|l|}{ Weight (kg) } \\
\hline Baseline & $81.4 \pm 2.90$ & $81.0 \pm 6.50$ & $72 \pm 4.25$ \\
\hline Treated & $84.7 \pm 3.0$ & $81.4 \pm 6.4$ & - \\
\hline \multicolumn{4}{|l|}{ BMI $\left(\mathrm{kg} / \mathrm{m}^{2}\right)$} \\
\hline Baseline & $28.4 \pm 2.8$ & $28.2 \pm 3.1$ & $24.7 \pm 1.5$ \\
\hline Treated & $30.0 \pm 2.7$ & $29.0 \pm 3.0$ & - \\
\hline \multicolumn{4}{|l|}{$\mathrm{HbA}_{1} \mathrm{c}(\%)$} \\
\hline Baseline & $7.21 \pm 0.29$ & $7.38 \pm 0.26$ & - \\
\hline Treated & $7.20 \pm 0.14$ & $7.29 \pm 0.32$ & - \\
\hline \multicolumn{4}{|l|}{ Fasting glucose $(\mathrm{mmol} / \mathrm{l})$} \\
\hline Baseline & $9.39 \pm 0.61 *$ & $9.54 \pm 0.57 *$ & $5.30 \pm 0.30$ \\
\hline Treated & $9.84 \pm 0.54 *$ & $7.36 \pm 0.46 \dagger$ & - \\
\hline \multicolumn{4}{|l|}{ Fasting cholesterol (mmol/l) } \\
\hline Baseline & $5.02 \pm 0.16$ & $5.16 \pm 0.15$ & $4.64 \pm 0.45$ \\
\hline Treated & $15 \pm 0.22$ & $4.70 \pm 0.33$ & - \\
\hline \multicolumn{4}{|l|}{ Fasting triglyceride $(\mathrm{mmol} / \mathrm{l})$} \\
\hline Baseline & $1.05(1.0,1.4)^{*}$ & $1.32(1.0,2.1)^{*}$ & $0.71(0.6,1.2)$ \\
\hline Treated & $1.30(1.0,1.6)^{*}$ & $0.89(0.40,1.3) \dagger$ & - \\
\hline \multicolumn{4}{|l|}{ Fasting NEFA $(\mu \mathrm{mol} / \mathrm{l})$} \\
\hline Baseline & $444 \pm 49$ & $466 \pm 61$ & $413 \pm 48$ \\
\hline Treated & $522 \pm 62$ & $437 \pm 64$ & - \\
\hline \multicolumn{4}{|l|}{ Fasting insulin (pmol/1) } \\
\hline Baseline & $71 \pm 9 *$ & $80 \pm 17 *$ & $43 \pm 4$ \\
\hline Treated & $94 \pm 13 * \ddagger$ & $58 \pm 10 \dagger$ & - \\
\hline \multicolumn{4}{|l|}{ HOMA insulin resistance } \\
\hline Baseline & $4.0 \pm 0.5^{*}$ & $5.1 \pm 1.2 *$ & - \\
\hline Treated & $6.0 \pm 0.8 *+$ & $3.0 \pm 0.5^{*} \dagger$ & $1.5 \pm 0.1$ \\
\hline
\end{tabular}


Table 2 Fasting plasma lipoprotein profile of the control subjects studied and of diabetic patients before and after treatment with pioglitazone or glibenclamide

\begin{tabular}{ccc} 
& Type 2 diabetic patients & Control subjects \\
\hline Glibenclamide group & Pioglitazone group &
\end{tabular}

\begin{tabular}{|c|c|c|c|}
\hline \multicolumn{4}{|c|}{ Total VLDL cholesterol (mmol/l) } \\
\hline Baseline & $0.52(0.46,0.98)$ & $0.56(0.51,0.65)$ & $0.45(0.39,0.64)$ \\
\hline Treated & $0.58(0.50,0.93)$ & $0.41(0.31,0.90)$ & - \\
\hline \multicolumn{4}{|c|}{ Triglyceride (mmol/l) } \\
\hline Baseline & $0.54(0.47,0.88)^{*}$ & $0.67(0.51,1.04)^{*}$ & $0.41(0.29,0.69)$ \\
\hline Treated & $0.50(0.45,0.87)$ & $0.35(0.20,0.85) \dagger$ & - \\
\hline \multicolumn{4}{|c|}{ VLDL-1 cholesterol (mmol/l) } \\
\hline Baseline & $0.27(0.22,0.52)$ & $0.29(0.24,0.36)$ & $0.24(0.18,0.41)$ \\
\hline Treated & $0.18(0.17,0.47)$ & $0.15(0.10,0.54)$ & - \\
\hline \multicolumn{4}{|c|}{ Triglyceride (mmol/l) } \\
\hline Baseline & $0.39(0.31,0.62)^{*}$ & $0.47(0.35,0.61)^{*}$ & $0.21(0.16,0.47)$ \\
\hline Treated & $0.31(0.27,0.63)^{*}$ & $0.24(0.11,0.63)^{*}$ & - \\
\hline \multicolumn{4}{|c|}{ VLDL-2 cholesterol (mmol/l) } \\
\hline Baseline & $0.27(0.23,0.48)$ & $0.29(0.21,0.35)$ & $0.23(0.19,0.26)$ \\
\hline Treated & $0.30(0.25,0.54)$ & $0.25(0.19,0.38)$ & - \\
\hline \multicolumn{4}{|c|}{ Triglyceride (mmol/l) } \\
\hline Baseline & $0.17(0.13,0.30)^{*}$ & $0.21(0.13,0.46)^{*}$ & $0.15(0.09,0.25)$ \\
\hline Treated & $0.18(0.16,0.27)^{*}$ & $0.13(0.08,0.23) \dagger$ & - \\
\hline \multicolumn{4}{|c|}{ IDL cholesterol (mmol/l) } \\
\hline Baseline & $0.83(0.75,1.28)$ & $0.94(0.82,1.05)$ & $0.57(0.36,0.99)$ \\
\hline Treated & $0.75(0.57,1.42)$ & $0.51(0.43,1.16)$ & - \\
\hline \multicolumn{4}{|c|}{ LDL cholesterol (mmol/l) } \\
\hline Baseline & $1.98(1.88,2.50)$ & $2.05(1.87,2.38)$ & $2.02(1.76,2.31)$ \\
\hline Treated & $1.56(1.47,2.08)$ & $1.70(1.41,2.11)$ & - \\
\hline \multicolumn{4}{|c|}{ HDL cholesterol (mmol/l) } \\
\hline Baseline & $1.19(0.99,1.63)^{*}$ & $1.07(0.98,1.27)^{*}$ & $1.31(1.11,1.84)$ \\
\hline Treated & $1.07(0.95,1.20)^{*}$ & $0.89(0.79,1.16)^{*}$ & - \\
\hline
\end{tabular}

Data for cholesterol content were normally distributed and are shown as means \pm SEM; data for triglyceride content were not normally distributed and are shown as medians $(95 \% \mathrm{CIs})$

${ }^{*} p<0.01$ (ANOVA) for difference between diabetic patients and control subjects; $\uparrow p<0.04$ (paired $t$-test) for difference between baseline and pioglitazone treatment. IDL Intermediate-density lipoprotein

Table 3 The overall postprandial disposal (AUC) of glucose, triglyceride and retinyl palmitate-containing lipoproteins (chylomicron and chylomicron remnants) in control subjects and type 2 diabetic patients before and after treatment with either pioglitazone or glibenclamide

$\begin{array}{lcc} & \text { Type 2 diabetic patients } & \text { Control subjects } \\ \text { Glibenclamide group } & \text { Pioglitazone group } & \end{array}$

\begin{tabular}{|c|c|c|c|}
\hline \multicolumn{4}{|c|}{ Glucose AUC $\left(\mathrm{mmol} \mathrm{l}^{-1} 8 \mathrm{~h}^{-1}\right)$} \\
\hline Baseline & $65.63 \pm 6.91 *$ & $76.67 \pm 4.08^{*}$ & $40.40 \pm 0.90$ \\
\hline Treated & $75.56 \pm 4.72 *$ & $61.70 \pm 6.17 * \dagger$ & - \\
\hline \multicolumn{4}{|c|}{ Triglyceride AUC $\left(\mathrm{mmol}^{-1} 8 \mathrm{~h}^{-1}\right)$} \\
\hline Baseline & $20.78 \pm 1.54^{*}$ & $21.11 \pm 2.32 *$ & $13.54 \pm 1.93$ \\
\hline Treated & $21.50 \pm 2.32 *$ & $14.18 \pm 2.28 \dagger$ & - \\
\hline \multicolumn{4}{|c|}{ Chylomicron retinyl palmitate AUC ( $\left.\mu \mathrm{mol} \mathrm{l}^{-1} 8 \mathrm{~h}^{-1}\right)$} \\
\hline Baseline & $21.02 \pm 7.51 *$ & $18.99 \pm 4.05^{*}$ & $12.56 \pm 1.29$ \\
\hline Treated & $22.28 \pm 4.96^{*}$ & $8.04 \pm 1.34 \dagger$ & - \\
\hline \multicolumn{4}{|c|}{ Chylomicron remnant retinyl palmitate AUC $\left(\mu \mathrm{mol} \mathrm{l} \mathrm{l}^{-1} 8 \mathrm{~h}^{-1}\right)$} \\
\hline Baseline & $23.32 \pm 3.69^{*}$ & $24.16 \pm 6.54 *$ & $11.69 \pm 2.65$ \\
\hline Treated & $20.97 \pm 3.67 *$ & $15.49 \pm 1.91$ & - \\
\hline
\end{tabular}

Data are means \pm SEM

${ }^{*} p<0.05$ (ANOVA) for difference between diabetic patients and control subjects; $\uparrow p<0.05$ (paired $t$-test) for difference between baseline and pioglitazone-treatment in diabetic patients 


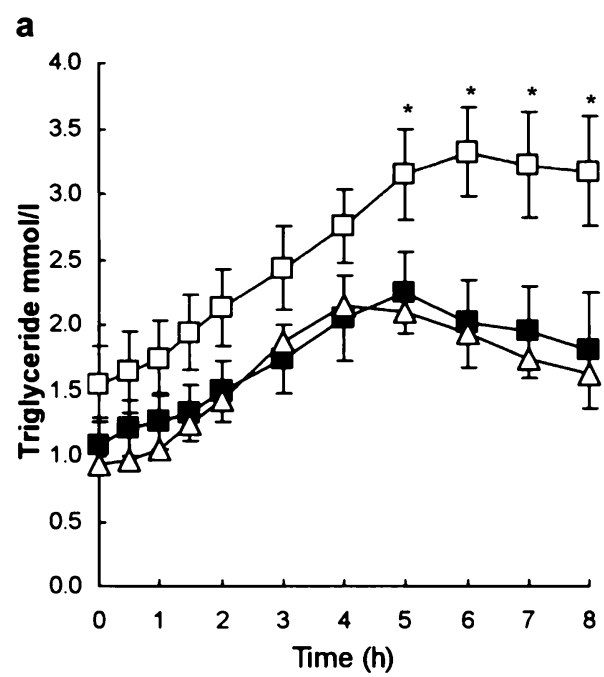

Fig. 1 Effect of the test meal on plasma triglyceride concentrations in control subjects and type 2 diabetic patients before and after treatment. a Treatment with pioglitazone; paired $t$-test analysis of pioglitazone-treated patients (solid squares) vs the same patients at baseline (open squares) showed a significant fall in triglyceride $(p<0.02)$ at all times; data for the untreated diabetic patients were significantly higher in the later postprandial period than in the control group (open triangles) or the same patients after pioglitazone

the decrease in VLDL-1 triglyceride narrowly failed to achieve statistical significance $(p<0.07)$. No changes were observed in the glibenclamide group.

There were no differences in intermediate-density lipoprotein and LDL cholesterol concentrations at baseline between diabetic patients and controls and no changes with either therapy. HDL cholesterol was significantly greater in the controls compared with the diabetic patients but treatment with neither drug made any difference.

\section{Postprandial glucose disposal}

Overall glucose disposals of the control and diabetic subjects, both untreated and treated, expressed as AUCs are given in Table 3. The significant fall in FPG observed after pioglitazone treatment is reflected in a smaller AUC for

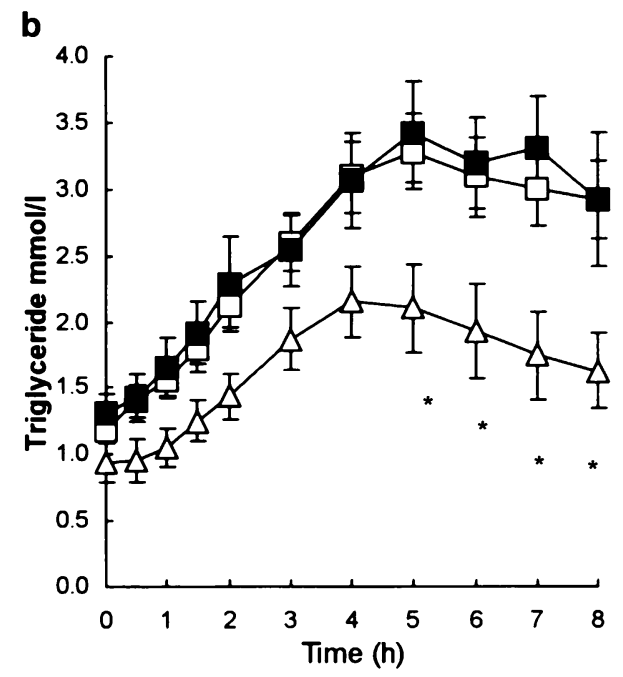

treatment $\left({ }^{*} p<0.05\right.$, ANOVA). b Treatment with glibenclamide; data for the glibenclamide-treated patients (solid squares) remained unaltered from the pretreatment levels (open squares) and were significantly greater that those of control subjects (open triangles) in the later postprandial period $(* p<0.05$, ANOVA); paired $t$-test analysis of the effect of glibenclamide treatment revealed no significant differences from baseline at any time. Data are means \pm SEM

glucose in response to the test meal. Postprandial glucose excursions exert the greatest influence on haemoglobin glycation and $\mathrm{HbA}_{1} \mathrm{c}$ levels were found to correlate with the postprandial AUCs for glucose both at baseline and after treatment (pioglitazone: baseline $r^{2}=0.41, p<0.01$; treated $r^{2}=0.36, p<0.01$; glibenclamide: baseline $r^{2}=0.44$, $p<0.01$; treated $r^{2}=0.62, p<0.01$ ). There were no significant relationships with the FPG in either group before or after treatment.

\section{Postprandial triglyceride disposal}

Postprandial plasma triglyceride disposal curves are shown in Fig. 1. In the controls, plasma triglycerides rose to a peak of approximately $1 \mathrm{mmol} / \mathrm{l}$ above the fasting levels after $4 \mathrm{~h}$ and thereafter declined. Diabetic patients in the untreated
Fig. 2 Postprandial disposal of retinyl palmitate in intact chylomicrons from control subjects (open triangles) and diabetic patients before (open squares) and after (solid squares) treatment with pioglitazone (a) or glibenclamide (b). Data are means \pm SEM $; * p<0.05$, untreated diabetic patients significantly greater than after treatment with pioglitazone (a); * $p<0.05$, control subject values significantly lower than baseline or glibenclamide-treated diabetic patients (b)
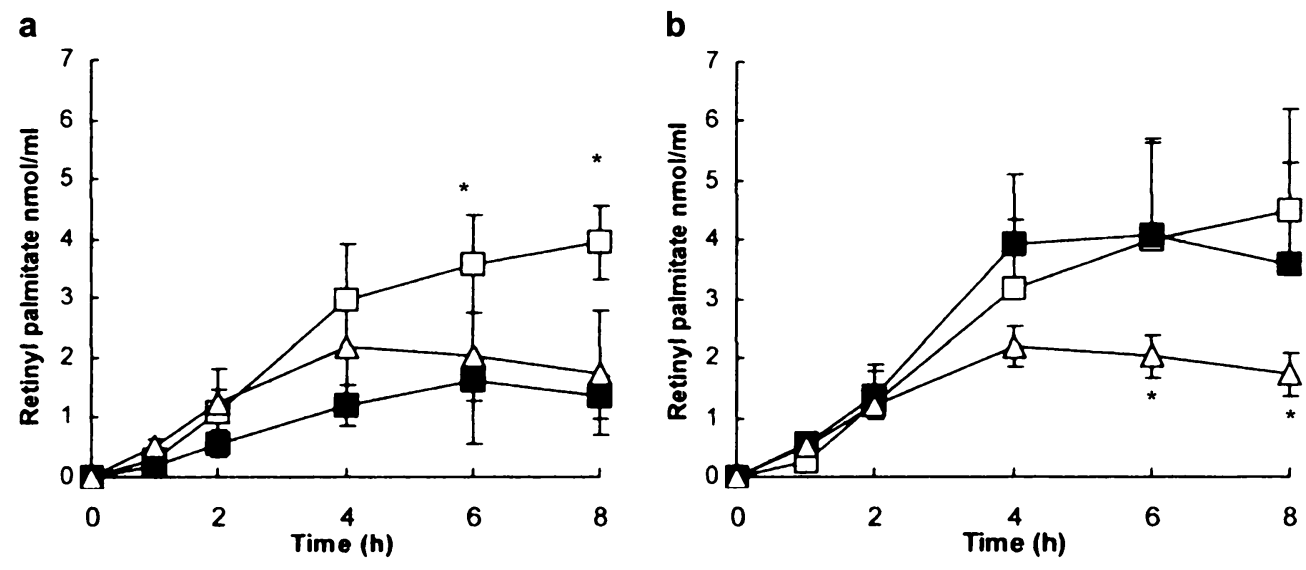
Fig. 3 Postprandial retinyl palmitate present in chylomicron-remnant-containing fractions from control subjects (open triangles) and type 2 diabetic patients before (open squares) and after (solid squares) treatment with either pioglitazone (a) or glibenclamide (b). Data are means \pm SEM; ${ }^{*} p<0.05$, untreated patients significantly greater than after treatment

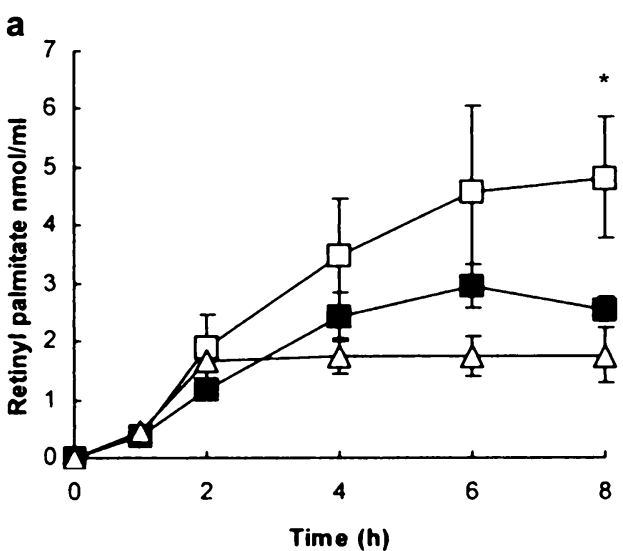

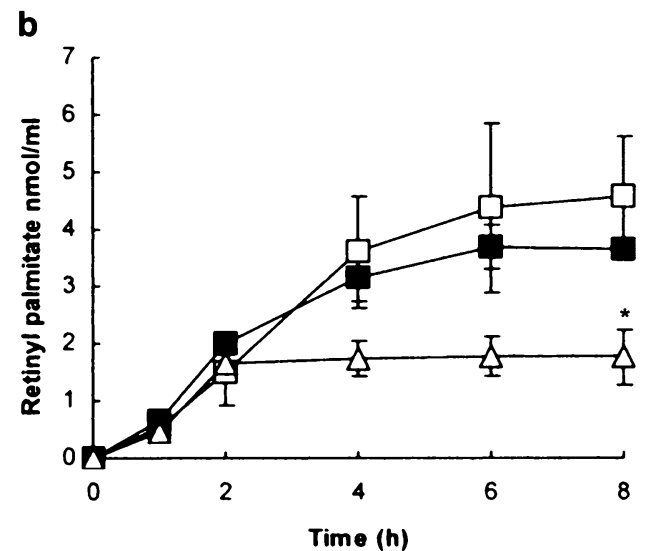

state showed the characteristically delayed and exaggerated postprandial lipaemia associated with type 2 diabetes with the peak triglyceride reaching approximately $1.8 \mathrm{mmol} / 1$ above the fasting levels after $4-5 \mathrm{~h}$. The effect of treatment with pioglitazone was to reduce postprandial lipaemia to levels seen in controls. This reduction was significant at all time points studied when the data were analysed by paired $t$-test, whereas glibenclamide treatment made no difference to the disposal of triglyceride. The overall disposal (AUC) of triglyceride is shown in Table 3; it can be seen that there is a reduction in the triglyceride excursion in the pioglitazone-treated patients that did not occur in the glibenclamide-treated group.

\section{$\mathrm{RP}$ disposal in chylomicron and chylomicron remnant fractions}

Disposal of chylomicron and chylomicron remnant RP are shown in Figs. 2 and 3. Pioglitazone treatment had a marked effect on chylomicron RP disposal, restoring concentrations to those seen in controls. Glibenclamide treatment made little quantitative or qualitative difference to the disposal of chylomicron RP.
Chylomicron remnant RP disposal was exaggerated and prolonged in both groups of diabetic subjects at baseline and had not peaked after $8 \mathrm{~h}$. Pioglitazone treatment restored disposal of chylomicron remnant RP to control levels. Glibenclamide treatment had no effect.

The AUCs for chylomicron and chylomicron remnant RP disposal in the diabetic patients at baseline and after treatment along with those of controls are shown in Table 3. These data support the findings for triglyceride disposal, in that overall insulin sensitisation has a beneficial effect on postprandial lipid disposal in contrast to sulphonylurea treatment, which showed no effects.

\section{Postprandial plasma NEFA level}

During the first $2 \mathrm{~h}$ postprandially, plasma NEFA levels fell due to the insulin-dependent suppression of adipose tissue lipase activity. In control subjects the lowest point in NEFA concentrations occurred significantly earlier than in the untreated diabetic patients (control $1.15 \pm 0.17 \mathrm{~h}, p<0.02$, ANOVA; baseline glibenclamide group $2.68 \pm 0.41 \mathrm{~h}$; baseline pioglitazone group $2.75 \pm 0.44 \mathrm{~h}$ ). Pioglitazone treatment shortened the period before the nadir in NEFA levels
Fig. 4 Hepatic lipase (a) and lipoprotein lipase (b) activities in post-heparin plasma from control subjects (open bars) and type 2 diabetic patients both before (shaded bars) and after (black bars) treatment with either pioglitazone or glibenclamide. Lipase activity is expressed as release of oleic acid in $\mu \mathrm{mol} \mathrm{h}{ }^{-1} \mathrm{ml} \mathrm{plasma}^{-1}$. Data are means \pm SEM; $* p<0.03$ (ANOVA), activity of control subjects significantly lower than that of diabetic patients before or after treatment with glibenclamide or pioglitazone;

$*_{*}^{*} p<0.005$, paired $t$-test analysis before and after treatment with pioglitazone a

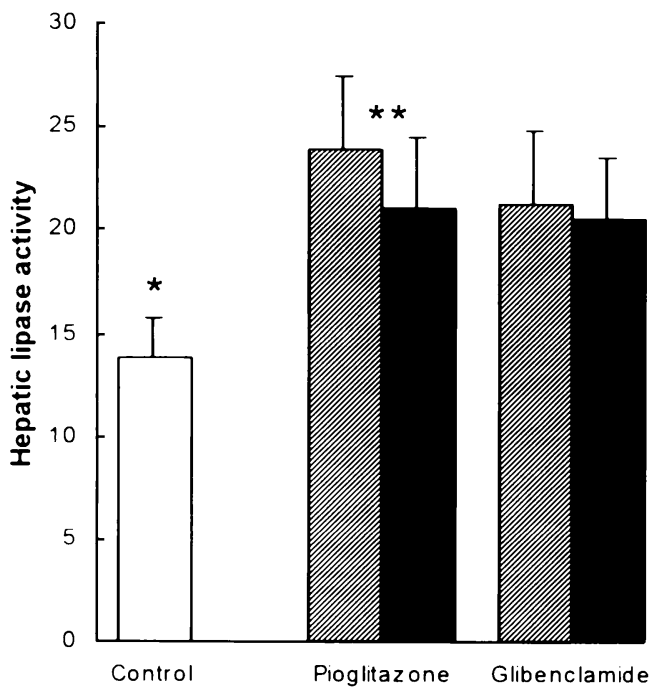

b

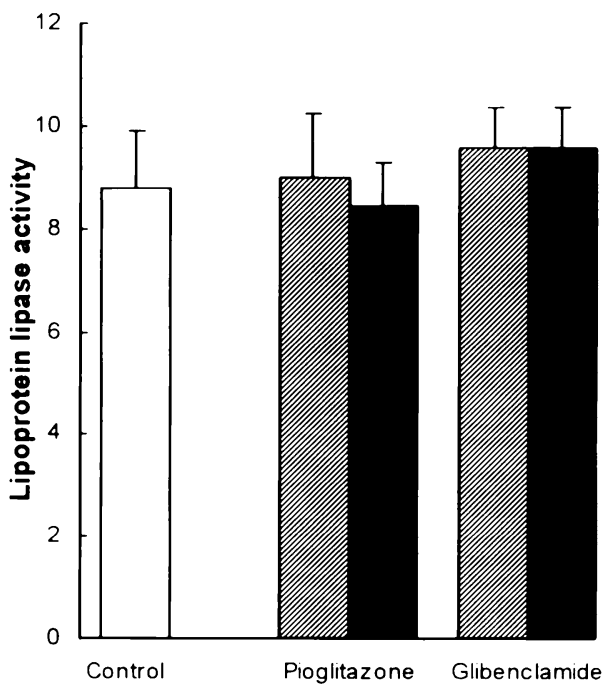


Table 4 Fasting and postprandial lecithin:cholesterol acyl transferase (LCAT), cholesteryl ester transfer protein (CETP) and phospholipid transfer protein (PLTP) activities in plasma from control subjects and type 2 diabetic patients before and after treatment with either glibenclamide or pioglitazone

\begin{tabular}{|c|c|c|c|c|c|c|}
\hline & \multicolumn{2}{|c|}{$\begin{array}{c}\text { LCAT activity } \\
(\mu \mathrm{mol} \text { cholesterol esterified } \\
\left.\mathrm{h}^{-1} 1 \text { plasma }^{-1}\right)\end{array}$} & \multicolumn{2}{|c|}{$\begin{array}{c}\text { CETP activity } \\
(\mu \mathrm{mol} \text { cholesterol ester transferred } \\
\left.\mathrm{h}^{-1} 1 \text { plasma }^{-1}\right)\end{array}$} & \multicolumn{2}{|c|}{$\begin{array}{c}\text { PLTP activity } \\
(\mu \mathrm{mol} \text { phospholipid transferred } \\
\left.\mathrm{h}^{-1} 1 \text { plasma }^{-1}\right)\end{array}$} \\
\hline & $0 \mathrm{~h}$ & $4 \mathrm{~h}$ & $0 \mathrm{~h}$ & $4 \mathrm{~h}$ & $0 \mathrm{~h}$ & $4 \mathrm{~h}$ \\
\hline $\begin{array}{l}\text { Control subjects } \\
\text { Pioglitazone }\end{array}$ & $43.7 \pm 3.3$ & $47.6 \pm 3.3$ & $8.8 \pm 1.1$ & $21.8 \pm 2.8^{* *}$ & $1.85 \pm 0.08$ & $1.61 \pm 0.08^{*}$ \\
\hline Baseline & $36.0 \pm 3.4$ & $50.7 \pm 4.9$ & $6.4 \pm 0.9$ & $20.7 \pm 3.0^{* *}$ & $1.55 \pm 0.07 \dagger$ & $1.44 \pm 0.09$ \\
\hline Treated & $39.3 \pm 4.2$ & $46.8 \pm 3.9$ & $9.1 \pm 1.5$ & $15.4 \pm 2.3 * *$ & $1.69 \pm 0.06$ & $1.53 \pm 0.07 *$ \\
\hline Glibenclamide & & & & & & $1.52 \pm 0.08$ \\
\hline Baseline & $51.5 \pm 4.0$ & $55.2 \pm 8.2$ & $10.5 \pm 2.9$ & $21.8 \pm 2.9^{* *}$ & $1.64 \pm 0.07 \dagger$ & \\
\hline Treated & $50.5 \pm 4.1$ & $48.8 \pm 6.3$ & $6.4 \pm 0.9$ & $20.7 \pm 3.0 * *$ & $1.59 \pm 0.10 \dagger$ & $1.51 \pm 0.09$ \\
\hline
\end{tabular}

Data are means \pm SEM

${ }^{*} p<0.01$ for decrease in PLTP activity $(0 \mathrm{~h}$ vs $4 \mathrm{~h})$, paired $t$-test; ${ }^{* *} p<0.002$ for increase in CETP activity $(0 \mathrm{~h}$ vs $4 \mathrm{~h})$, paired $t$-test; $\dagger p<0.05$ for difference from control subjects

was reached to that of control subjects, from which it no longer differed significantly (pioglitazone-treated $1.80 \pm$ $0.41 \mathrm{~h}$; control $1.15 \pm 0.17 \mathrm{~h}, p=\mathrm{NS}$ ), whereas glibenclamide treatment had no effect on the nadir in NEFA concentrations that still occurred significantly later than in control subjects (glibenclamide-treated $2.31 \pm 0.41 \mathrm{~h}$; control 1.15 \pm $0.17 \mathrm{~h}, p<0.02)$. However, apart from this qualitative difference, paired $t$-test analysis showed no significant quantitative effects of either pioglitazone or glibenclamide treatment on fasting and postprandial NEFA when compared with the untreated diabetic subjects and controls.

Hepatic and LPL activities

Hepatic lipase and LPL activities in post-heparin plasma from controls and patients before and after treatment with glibenclamide or pioglitazone are shown in Fig. 4. These measurements, made in the presence of substrate excess, reflect the mass of enzyme but not necessarily the activity in situ. Hepatic lipase activities were higher in both groups of diabetic patients than in controls $(p<0.03$, ANOVA). Pioglitazone treatment caused a small, $\cong 15 \%$, but highly significant fall in hepatic lipase activity $(p<0.0003)$ when analysed by the paired $t$-test that did not occur in the glibenclamide-treated patient group $(p>0.5)$. LPL activities were similar in the control and the diabetic patients, irrespectively of the treatment regimen employed.

Fasting and postprandial neutral lipid synthesis and exchange

Cholesteryl ester synthesis by LCAT and its transfer from HDL by CETP was measured in plasma sampled in the fasting state and $4 \mathrm{~h}$ after the meal. The results are shown in Table 4, along with the PLTP activity. In the fasting state no differences were found in the activity of LCAT between the diabetic patient controls either at the baseline or following pioglitazone or glibenclamide. The test meal made no impact.

CETP activity increased significantly postprandially but no significant differences were found between the activity expressed in control and diabetic patients, whether or not treated with either pioglitazone or glibenclamide.

PLTP activity was greater in controls than in the diabetic patients. Four hours after the test meal there was a significant fall in PLTP activity in controls when compared with fasting activity. In the baseline state, diabetic patients also showed a postprandial fall in activity but this did not achieve statistical significance in either group. Treatment with pioglitazone re-established the statistical significance of the postprandial decrease in PLTP activity to the control situation while glibenclamide treatment had no effect.

\section{Discussion}

Previous studies, including our own, pointed to important correlations between insulin sensitivity and lipid and lipoprotein metabolism $[4,22]$. In this, the first study on the influence of the TZD pioglitazone on postprandial metabolism, potentially important changes in glucose, lipid and lipoprotein metabolism are reported.

The improvement in fasting triglyceride after pioglitazone treatment was caused by a significant decrease in the amount of triglyceride present in VLDL, particularly in the smaller VLDL-2 species, and although VLDL-1 triglyceride also fell, this did not achieve statistical significance. Since there were no changes in the cholesterol present in VLDL, it seems reasonable that improved fasting triglyceride involves a reduction in hepatic triglyceride synthesis or its integration into apo-B100, both processes being influenced by insulin [28, 29].

The mechanism for the reduction in hepatic triglyceride synthesis is thought to involve decreased insulin-dependent adipose tissue lipolysis that results in a lowering of the 
plasma NEFA concentration. This in turn is proposed to influence the glucose-fatty acid cycle (Randle cycle) by promoting skeletal muscle glucose oxidation, while simultaneously restricting the supply of NEFA to the liver as substrate for hepatic triglyceride synthesis [30]. However, given that glycaemic control was maintained at pretreatment levels in this study, and that we found only a trend for plasma NEFA levels to be reduced by pioglitazone treatment, other mechanisms for the fall in hepatic triglyceride synthesis involving the direct action of pioglitazone on hepatic PPAR- $\gamma$ cannot be ruled out [31]. Alternatively, a decrease in hepatic triglyceride synthesis could be indirectly brought about by pioglitazone stimulation of adipocyte PPAR- $\gamma$ to increase the secretion of cytokines, such as adiponectin, which then act on the liver to reduce insulin resistance [32].

In the postprandial state, pioglitazone treatment reduced both the extent and duration of the triglyceride excursion following the test meal to levels seen in control subjects. This was apparently due to a more rapid disposal of chylomicrons and the chylomicron remnants. We used the well-established technique of oral RP labelling of chylomicrons. This method can be criticised because of the exchange of RP with other triglyceride-rich lipoproteins of hepatic origin occurring in the late postprandial phase, but this would not be expected to influence the results of our studies, which were limited to $8 \mathrm{~h}$ [33]. The improvement in triglyceride and chylomicron metabolism after pioglitazone treatment of the patients is likely to be due to a reduction in insulin resistance effected by PPAR- $\gamma$ activation rather than to an overall improvement in mechanisms directly initiated by insulin, since insulin-providing therapy with glibenclamide had no effect.

The possible causes that give rise to abnormal postprandial lipaemia include abnormal chylomicron composition and their secretion into the circulation, and abnormal processing of intestinal lipoproteins by lipases and lipid transfer proteins as well as abnormal hepatic uptake of triglyceride-rich lipoproteins. The synthesis of chylomicrons with increased triglyceride content has been reported in an animal model of insulin resistance and this abnormality is corrected by treatment with TZD [34]. While normalisation of the composition of chylomicrons after pioglitazone treatment may provide, in part, an explanation of our findings of improved triglyceride disposal, other factors, such as reduced competition between hepatic and intestinal lipoproteins for lipolytic enzymes and transfer proteins brought about by reduced VLDL synthesis and improved uptake of triglyceride-rich lipoprotein remnants, could also be involved, given that delayed triglyceride disposal is evident in the later postprandial period.

We found no evidence to support reduced activity of LPL as a mechanism for the exaggerated triglyceride excursion seen in type 2 diabetes. Enzyme activity measured in vitro was present to the same extent as in controls and was unaffected by insulin sensitisation or glibenclamide treatment, which argues against a role of PPAR- $\gamma$ or a direct influence of insulin in LPL biosynthesis. However, LPL activity in vivo is modulated by apolipoproteins C-II and C-III present on lipoproteins. Apolipoprotein C-III is an inhibitor of LPL and its absence is known to accelerate the disposal of dietary lipid [35]. A recent study on the effect of treatment with pioglitazone in a group of eight type 2 diabetic patients has shown a decrease in apolipoprotein C-III levels, although this is not thought to be mediated through the PPAR- $\gamma$ receptor [36]. Some influence of insulin resistance on C-III levels can be inferred from the finding that its concentration in plasma correlates strongly with the level of production and concentration of VLDL triglyceride and also with the HOMA score of insulin sensitivity [37]. Taking these findings together, it remains possible that attenuated lipolysis by LPL of chylomicrons and other triglyceride-rich lipoproteins is the mechanism that explains delayed disposal of dietary lipid, even though the LPL activities measured under optimal conditions were equal in the control subjects and the diabetic patients regardless of the treatment regime. Treatment with pioglitazone may have important effects on the availability of cofactors necessary for LPL activity in vivo rather than on the enzyme per se.

Hepatic lipase activity is known to be greater in type 2 diabetes [38]. Our results confirm this finding, but we also report a significant decrease in hepatic lipase activity after pioglitazone treatment but not with glibenclamide. It seems likely that the mechanism of this effect is associated with reduced insulin resistance through PPAR- $\gamma$, since glibenclamide, which has been shown to have no effect on insulin sensitivity, did not change hepatic lipase activity. However, reduced hepatic lipase could be an indirect consequence of insulin sensitisation stemming from the pioglitazoneinduced reduction in postprandial lipaemia. In the pathogenesis of type 2 diabetes it is thought that increased hepatic lipase activity is responsible for the so-called atherogenic LDL size profile, in which there is a predominance of small dense particles caused by disproportionate hepatic lipase lipolysis of LDL triglyceride [38]. We did not measure LDL particle size directly, but it is claimed that the AIP is a surrogate measure for the presence of small dense LDL particles and we found that pioglitazone treatment caused an improvement in this index [39].

As previously reported, LCAT and CETP activities did not differ between diabetic patients and controls and did not change with pioglitazone or glibenclamide [22]. Similarly to the CETP determinations, PLTP activity was measured utilising endogenous lipoproteins as donors and acceptors of phospholipid, providing a measurement of the ambient transfer rather than the mass of protein present. In this study we have found that overall phospholipid transfer tended to be lower in the diabetic subjects than in controls, but whereas cholesteryl ester transfer increased postprandially phospholipid transfer decreased. The postprandial fall in PLTP activity is unexplained but likely be related to insulin sensitivity [40-43].

We attempted to keep glycated haemoglobin concentrations similar in both treated groups in order to determine whether effects on lipid metabolism were independent of glycaemic control. Overall, we achieved this, but in the pioglitazone group we observed a reduction in FPG and 
postprandial glycaemia. We cannot therefore conclude categorically that the effects on lipid metabolism seen with pioglitazone are independent of glycaemic effects. However, in a previous study, glibenclamide did not have an effect on postprandial lipid metabolism after 12 weeks of therapy, despite significant reductions in fasting and postprandial glucose as well as $\mathrm{HbA}_{1} \mathrm{c}$ [15]. In our study, moreover, glibenclamide, as expected, significantly increased fasting insulin levels and the HOMA score. Therefore it is likely, but not conclusive, that the differences in lipid metabolism reported here are due to the differing mechanisms of actions of the two drugs.

Significant quantitative changes in fasting plasma NEFA were not observed with insulin sensitisation, although the diabetic patients showed a trend towards higher NEFA levels than controls, this effect being lowered by pioglitazone treatment. Insulin sensitisation with pioglitazone partially corrected the delayed decrease in postprandial NEFA levels seen in diabetic patients, suggesting an effect of insulin sensitisation on the inhibition of adipocyte NEFA release $[44,45]$. In our study, pioglitazone reduced fasting triglyceride levels; however, this does not seem to be the case with rosiglitazone, which has been shown to enhance postprandial triglyceride disposal, with this effect being attributed to an improvement in the lipolysis of intact chylomicrons [46]. The reason for the differences in action of these two TZDs is not known, but may lie in the fact that pioglitazone reportedly has a greater potential than rosiglitazone to react with the PPAR- $\alpha$ receptor, but other differences in their pleiotropic effects or actions not involving nuclear genes cannot be ruled out [47-50]. After treatment with pioglitazone, we have also shown a significant improvement in the disposal of chylomicrons, and also in chylomicron remnant metabolism, which, coupled with the reduction in fasting VLDL and triglyceride, is responsible for a $35 \%$ improvement in postprandial triglyceride clearance.

We conclude that insulin sensitisation with pioglitazone has major effects in restoring postprandial lipaemia to normal, while also correcting fasting hypertriglyceridaemia; both of these factors may have affect atherogenic risk in people with diabetes.

Acknowledgement We would like to thank Mrs Céline Leseur for determination of PLTP activity.

\section{References}

1. Krentz AJ (2003) Lipoprotein abnormalities and their consequences for patients with type diabetes. Diabetes Obes Metab 5 (Suppl 1):S19-S27

2. Duvillard L, Pont F, Florentin E, Galland-Jos C, Gambert P, Verges B (2000) Metabolic abnormalities of apolipoprotein Bcontaining lipoproteins in non-insulin-dependent diabetes: a stable isotope kinetic study. Eur J Clin Invest 30:685-694

3. Koba S, Hirano T, Murayama S et al (2003) Small dense LDL phenotype is associated with postprandial increases of large VLDL and remnant-like particles in patients with acute myocardial infarction. Atherosclerosis 170:131-140
4. Cooper MB, Tan KC, Hales CN, Betteridge DJ (1996) Postprandial lipid metabolism and beta-cell function in noninsulin-dependent (type 2 diabetes mellitus) after a mixed meal with a high fat content. Diabet Med 13:816-827

5. Mero N, Syvanne M, Taskinen M-R (1998) Postprandial lipid metabolism in diabetes. Atherosclerosis 141 (Suppl 1):S53-S55

6. van Wijk JP, Halkes CJ, Erkelens DW, Castro-Cabezas M (2003) Fasting and daylong triglycerides in obesity with and without type 2 diabetes. Metabolism 52:1043-1049

7. Brunzell JD, Hazzard WR, Porte D Jr, Bierman EL (1973) Evidence for a common saturable triglyceride removal mechanism for chylomicrons and very low density lipoproteins in man. J Clin Invest 52:1578-1585

8. Riemens S, van-Tol A, Sluiter W, Dullaart R (1998) Elevated plasma cholesteryl ester transfer in NIDDM: relationships with apolipoprotein B-containing lipoproteins and phospholipid transfer protein. Atherosclerosis 140:71-79

9. Semenkovich CF (2004) Fatty acid metabolism and vascular disease. Trends Cardiovasc Med 14:72-76

10. Reaven GM (2002) Multiple CHD risk factors in type 2 diabetes: beyond hyperglycaemia. Diabetes Obes Metab 4 (Suppl 1):S13-S18

11. Lebovitz HE, Banerji MA (2001) Insulin resistance and its treatment by thiazolidinediones. Recent Prog Horm Res $56: 265-294$

12. Olefsky JM, Saltiel AR (2000) PPAR $\gamma$ and the treatment of insulin resistance. Trends Endocrinol Metab 11:362-368

13. Desvergne B, Wahli W (1999) Peroxisome proliferatoractivated receptors: nuclear control of metabolism. Endocr Rev 20:649-688

14. Fischer S, Patzak A, Rietzsch H et al (2003) Influence of treatment with acarbose or glibenclamide on insulin sensitivity in type 2 diabetic patients. Diabetes Obes Metab 5:38-44

15. Vakkilainen J, Mero N, Schweizer A, Foley JE, Taskinen MR (2002) Effects of nateglinide and glibenclamide on postprandial lipid and glucose metabolism in type 2 diabetes. Diabetes Metab Res Rev 18:484-490

16. Fletcher CD, Barnes JF, Farish E (1994) A rapid semi-micro method for the separation of lipoprotein fractions that uses a benchtop ultracentrifuge. Clin Chim Acta 226:95-99

17. Cathcart S, Dominiczak MH (1990) The measurement of lipoprotein subfractions in plasma using a tabletop ultracentrifuge. Ann Clin Biochem 27:459-464

18. Leonhardt W, Pietzsch J, Nitzsche S (1994) Very-fast ultracentrifugation of human plasma lipoproteins: influence of the centrifugal field on lipoprotein composition. Clin Chim Acta $224: 21-32$

19. Mackness MI, Durrington PN (1992) Lipoprotein separation and analysis for clinical studies. In: Converse CA, Skinner ER (eds) Lipoprotein analysis - a practical approach. IRL, Oxford, pp $1-42$

20. Demacker PN, Hijmans AG, Vos-Janssen HE, van't-Laar A, Jansen AP (1980) A study of the use of polyethylene glycol in estimating cholesterol in high-density lipoprotein. Clin Chem 26:1775-1779

21. De Ruyter MG, De Leenheer AP (1978) Simultaneous determination of retinol and retinyl esters in serum or plasma by reversed-phase high-performance liquid chromatography. Clin Chem 24:1920-1923

22. Tan KCB, Cooper MB, Ling KLE et al (1995) Fasting and postprandial determinants for the occurrence of small dense LDL species in non-insulin-dependent diabetic patients with or without hypertriglyceridaemia. Atherosclerosis 113:273-287

23. Bouly M, Masson D, Gross B et al (2001) Induction of the phospholipid transfer protein gene accounts for the high density lipoprotein enlargement in mice treated with fenofibrate. J Biol Chem 276:25841-25847

24. Henderson AD, Richmond W, Elkeles RS (1993) Hepatic and lipoprotein lipases selectively assayed in postheparin plasma. Clin Chem 39:218-223 
25. Matthews DR, Hosker JP, Rudenski AS, Naylor BA, Treacher DF, Turner RC (1985) Homeostasis model assessment: insulin resistance and beta-cell function from fasting plasma glucose and insulin concentrations in man. Diabetologia 28:412-419

26. Dobiasova M, Frolich J (2001) The plasma parameter $\log (\mathrm{TG} /$ HDL-C) as an atherogenic index: correlation with lipoprotein particle size and esterification rate in apoB-lipoprotein-depleted plasma $\left(\mathrm{FER}_{\mathrm{HDL}}\right)$. Clin Biochem 34:583-588

27. Bland JM, Altman DG (1996) Transformations means and confidence intervals. Br Med J 312:1079-1080

28. Brown AM, Gibbons GF (2001) Insulin inhibits the maturation phase of VLDL assembly via a phosphoinositide 3-kinasemediated event. Arterioscler Thromb Vasc Biol 21:1656-1661

29. Carpentier A, Taghibiglou C, Leung N et al (2002) Ameliorated hepatic insulin resistance is associated with normalization of microsomal triglyceride transfer protein expression and reduction in very low density lipoprotein assembly and secretion in the fructose-fed hamster. J Biol Chem 277:28795-28802

30. Frayn KN (2003) The glucose-fatty acid cycle: a physiological perspective. Biochem Soc Trans 31:1115-1119

31. Michael MD, Kulkarni RN, Postic C et al (2000) Loss of insulin signaling in hepatocytes leads to severe insulin resistance and progressive hepatic dysfunction. Mol Cell 6:87-97

32. Maeda N, Takahashi M, Funahashi T et al (2001) PPAR $\gamma$ ligands increase expression and plasma concentrations of adiponectin an adipose-derived protein. Diabetes 50:20942099

33. Krasinski SD, Cohn JS, Russel RM, Schaefer EJ (1990) Postprandial plasma vitamin A metabolism in humans: a reassessment of the use of plasma retinyl ester as marker for intestinally derived chylomicrons and their remnants. Metabolism 39:357-365

34. Leung N, Naples M, Uffelman K, Szeto L, Adeli K, Lewis GF (2004) Rosiglitazone improves intestinal lipoprotein in the fatfed Syrian Golden hamster. Atherosclerosis 174:235-241

35. Jong MC, Rensen PC, Dahlmans VE, van der Boom H, van Berkel TJ, Havekes LM (2001) Apolipoprotein C-III deficiency accelerates triglyceride hydrolysis by lipoprotein lipase in wildtype and apoE knockout mice. J Lipid Res 42:1578-1585

36. Nagashima K, Lopez C, Donovan D et al (2005) Effects of the PPAR $\gamma$ agonist pioglitazone on lipoprotein metabolism in patients with type 2 diabetes mellitus. J Clin Invest 115:13231332

37. Cohn JS, Patterson BW, Uffelman KD, Davignon J, Steiner G (2004) Rate of production of plasma and very-low-density lipoprotein (VLDL) apolipoprotein C-III is strongly related to the concentration and level of production of VLDL triglyceride in male subjects with different body weights and levels of insulin sensitivity. J Clin Endocrinol Metab 89:3949-3955
38. Jansen H, Verhoeven AJM, Sijbrands EJC (2002) Hepatic lipase: a pro- or anti-atherogenic protein? J Lipid Res 43:13521362

39. Tan MH, Johns D, Glazer NB (2004) Pioglitazone reduces atherogenic index of plasma in patients with type 2 diabetes. Clin Chem 50:1184-1188

40. Dullaart RP, van Tol A (2001) Short-term acipimox decreases the ability of plasma from type 2 diabetic patients and healthy subjects to stimulate cellular cholesterol efflux: a potentially adverse effect on reverse cholesterol transport. Diabet Med 18:509-513

41. Riemens SC, van Tol A, Sluiter WJ, Dullaart RP (1998) Plasma phospholipid transfer protein activity is related to insulin resistance: impaired acute lowering by insulin in obese type II diabetic patients. Diabetologia 41:929-934

42. Iris JAM, Jonkers IJ, Smelt A-H et al (2003) Decreased PLTP mass but elevated PLTP activity linked to insulin resistance in HTG: effects of bezafibrate therapy. J Lipid Res 44:1462-1469

43. Riemens SC, van-Tol A, Sluiter WJ, Dullaart RP (1999) Plasma phospholipid transfer protein activity is lowered by $24-\mathrm{h}$ insulin and acipimox administration: blunted response to insulin in type 2 diabetic patients. Diabetes 48:1631-1637

44. Van Harmelen V, Reynisdottir S, Cianflone K et al (1999) Mechanisms involved in the regulation of free fatty acid release from isolated human fat cells by acylation-stimulating protein and insulin. J Biol Chem 274:18243-18251

45. Tordjman J, Chauvet G, Quette J, Beale EG, Forest C, Antoine B (2003) Thiazolidinediones block fatty acid release by inducing glyceroneogenesis in fat cells. J Biol Chem 278 : 18785-18790

46. van Wijk JPH, de Koning EJP, Cabezas MC, Rabelink TJ (2005) Rosiglitazone improves postprandial triglyceride and FFA metabolism. Diabetes Care 28:844-849

47. Sakamoto J, Kimura H, Moriyama S et al (2000) Activation of human peroxisome proliferator-activated receptor (PPAR) subtypes by pioglitazone. Biochem Biophys Res Commun 278:704-711

48. Tan GD, Fielding BA, Currie JM et al (2005) The effects of rosiglitazone on fatty acid and triglyceride metabolism in type 2 diabetes. Diabetologia 48:83-95

49. Narce M, Poisson J-P (2003) Novel PPAR $\gamma$-dependent and independent effects for thiazolidinediones. Curr Opin Lipidol 14:651-652

50. Akbiyik F, Ray DM, Gettings KF, Blumberg N, Francis CW, Phipps RP (2004) Human bone marrow megakaryocytes and platelets express PPAR $\gamma$, and PPAR $\gamma$ agonists blunt platelet release of CD40 ligand and thromboxanes. Blood 104:13611368 